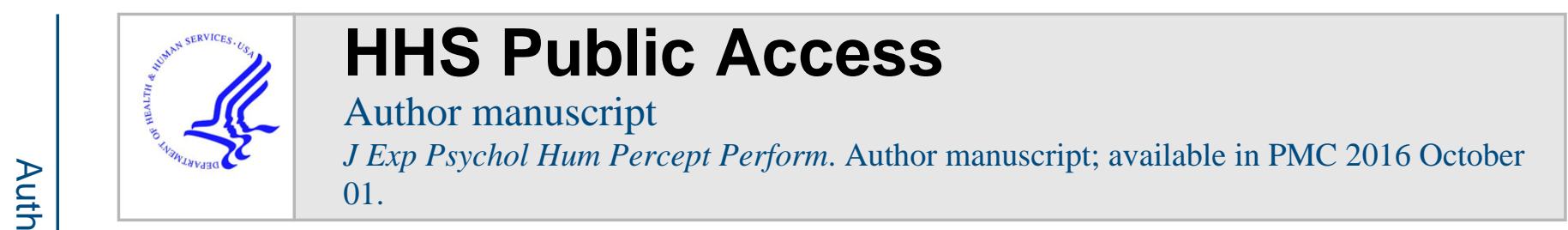

Published in final edited form as:

J Exp Psychol Hum Percept Perform. 2015 October ; 41(5): 1409-1419. doi:10.1037/xhp0000072.

\title{
Readers extract character frequency information from nonfixated-target word at long pre-target fixations during Chinese reading
}

\author{
Guojie Ma ${ }^{1,2}$, Xingshan $\mathbf{L i}^{1}$, and Keith Rayner ${ }^{3}$ \\ ${ }^{1}$ Key Laboratory of Behavioral Science, Institute of Psychology, Chinese Academy of Sciences \\ 2 University of Chinese Academy of Sciences, Beijing, China \\ ${ }^{3}$ Department of Psychology, University of California, San Diego
}

\begin{abstract}
We performed two eye movement studies to explore whether readers can extract character or word frequency information from nonfixated-target words in Chinese reading. In Experiments 1A and $1 \mathrm{~B}$, we manipulated the character frequency of the first character in a two-character target word and the word frequency of a two-character target word, respectively. We found that fixation durations on the pre-target words were shorter when the first character of a two-character target word was presented with high frequency. Such effects were not observed for word frequency manipulations of a two-character target word. In particular, further analysis revealed that such effects only occurred for long pre-target fixations. These results for character and word frequency manipulations were replicated in a within-subjects design in Experiment 2. These findings are generally consistent with the notion that characters are processed in parallel during Chinese reading. However, we did not find evidence that words are processed in parallel during Chinese reading.
\end{abstract}

\section{Keywords}

Chinese reading; eye movements; parafoveal-on-foveal effect; character processing; word processing

\footnotetext{
Some studies on reading in English have shown that the properties of a parafoveal word affect how long readers look at the currently fixated word (a parafoveal-on-foveal effect) (Kennedy, 1998). The possible existence of a lexical parafoveal-on-foveal effect has been used to differentiate two types of theories regarding whether words are processed in parallel or in serial during the reading of alphabetic languages (see Schotter, Angele, \& Rayner, 2012 for review). This same question is even more interesting in Chinese. There are no explicit markers (like spaces in English) to mark word boundaries in Chinese text (Li, Liu, \& Rayner, 2011; Li, Rayner, \& Cave, 2009; Hoosain, 1991), and words are closer to each other in Chinese sentences compared to English sentences. Thus, Chinese readers may
} 
extract more parafoveal information during reading. In such an instance, the lexical properties of nonfixated words in the parafovea would be more likely to influence the processing of the currently fixated word. In this study, our main objective was to examine whether lexical properties, such as word frequency or character frequency, would show a parafoveal-on-foveal effect during Chinese reading.

Parafoveal-on-foveal effects have long been taken as evidence supporting the parallel gradient hypothesis of attention allocation. According to this hypothesis, attention is spatially distributed to multiple words in the perceptual span, and multiple words can be processed simultaneously (Engbert \& Kliegl, 2011). Therefore, the lexical properties of parafoveal words can influence online foveal processing. Two typical parallel models of eye movement control are the SWIFT model (Engbert, Nuthmann, Richter, \& Kliegl, 2005) and the Glenmore model (Reilly \& Radach, 2006). For example, lexical parafoveal-on-foveal effects are expected in the SWIFT model because it assumes that viewing of the foveal word is influenced by both foveal and parafoveal processing (Kliegl, Risse, \& Laubrock, 2007). When the parafoveal information is difficult to process, the time of initiating a saccade from foveal fixation is delayed (resulting in an increased duration of fixation).

In contrast, lexical parafoveal-on-foveal effects are not usually predicted by the sequential attention shift hypothesis of attention allocation. Such models assume that words are processed one by one in a strictly serial order. Once the currently attended word $n$ (word $n$ indicates the currently fixated word, word $\mathrm{n}+1$ is the next and so on) has been completely processed, attention shifts to word $n+1$ (Reichle, Liversedge, Pollatsek, \& Rayner, 2009). A typical sequential model is the E-Z Reader model (Reichle, Pollatsek, Fisher, \& Rayner, 1998; Reichle, Rayner, \& Pollatsek, 2003). In the E-Z Reader model, words are processed serially from left to right, so the lexical properties of parafoveal words should not affect processing of the currently fixated word. Thus, sequential models do not predict lexical parafoveal-on-foveal effects.

Previous studies on alphabetic languages have yielded consistent orthographic parafovealon-foveal effects (Inhoff, Starr, \& Shindler, 2000; Kennedy, 1998, 2000, 2008; Kennedy, Pynte, \& Ducrot, 2002; Pynte, Kennedy, \& Ducrot, 2004; Starr \& Inhoff, 2004; White, 2008). Lexical parafoveal-on-foveal effects, however, have not been consistently observed (see Schotter et al., 2012 for review). The evidence supporting parafoveal-on-foveal effects of word frequency comes from corpus analysis (Kennedy \& Pynte, 2005; Kliegl, 2007) and non-reading tasks (Kennedy, 1998, 2000; Kennedy et al., 2002). Some of these studies have demonstrated that the fixation duration on the pre-target word was longer when the target words were low frequency (Kennedy \& Pynte, 2005), but other studies have reported the opposite direction of parafoveal-on-foveal effects (Kennedy, 1998, 2000). Moreover, parafoveal-on-foveal effects of word frequency have not been observed in many experimental studies (Henderson \& Ferreira, 1993; Rayner, Fischer, \& Pollatsek, 1998; White, 2008). Henderson and Ferreira (1993) found that neither the lexical frequency nor a combination of syntactic class, lexical frequency and length affected fixation durations on pre-target words. Based on these results, they argued that fixation measures reflected foveal instead of parafoveal processing. White (2008) found that only orthographic familiarity, and not word frequency of the target word, influenced fixation durations on pre-target words. 
Therefore, experimental studies have produced no strong evidence supporting parafovealon-foveal effects of word frequency in alphabetic languages.

In Chinese, lexical parafoveal-on-foveal effects have been observed in many experimental studies (Bai, Hu, \& Yan, 2009; Cui, Wang, Yan, \& Bai, 2010; Cui et al., 2013; M. Yan, Kliegl, Shu, Pan, \& Zhou, 2010; M. Yan, Richter, Shu, \& Kliegl, 2009; M. Yan \& Sommer, 2015; Yang, Wang, Xu, \& Rayner, 2009). Importantly, many studies have revealed that parafoveal word frequency affects fixation duration on pre-target words (Bai et al., 2009; Cui et al., 2010; M. Yan et al., 2010). M. Yan et al. (2010) found that the word frequency of word $n+1$ (a single-character word) could affect fixation duration on word n. Fixation durations were shorter when word $n+1$ was high frequency versus low frequency. It should be noted that the target words in their studies were single-character words. Because a singlecharacter word is also a character and word frequency has a high correlation (approximately .95) with character frequency, it is not clear whether these effects should be attributed to character frequency or word frequency.

In another Chinese study, Bai et al. (2009) used two-character words as target words to examine whether the frequency and the stroke numbers of non-fixated words (word $n+1$ ) affected fixation duration on the currently fixated word (word $\mathrm{n}$ ). They found that fixation durations on the fixated words were shorter when the non-fixated parafoveal words were high-frequency words versus low-frequency words. However, the frequency of the first characters of the two-character target words was not matched in their study. Because highfrequency two-character words tend to have high-frequency first characters (correlation coefficient: .163, $p<.001$ ), it is unknown whether the parafoveal-on-foveal effects of word frequency of two-character words could be attributed to the frequency of the first character. In addition, when we reanalyzed the second experiment from a previous study performed by Wei, Li, and Pollatsek (2013) ${ }^{1}$, we found no evidence for parafoveal-on-foveal effects of word frequency. Finally, in a corpus analysis reported by Li, Bicknell, Liu, Wei, and Rayner (2014), the frequency of the character in the parafovea affected fixation durations on the foveal word, but the frequency of the word in the parafovea did not. Therefore, the extent to which word frequency affects fixation duration on pre-target words in Chinese reading remains controversial. The evidence supporting an effect of word frequency in previous studies (Bai et al., 2009; M. Yan et al., 2010) may reflect parallel character processing instead of parallel word processing in Chinese reading.

In summary, previous studies on Chinese reading have observed parafoveal-on-foveal effects of single-character (M. Yan et al., 2010) and two-character (Bai et al., 2009) word frequency; however, these effects may actually be attributable to character frequency. Previous studies have not determined whether character frequency or word frequency in the parafoveal position can affect fixation duration on the currently fixated word. Because determining whether multiple words instead of characters can be processed simultaneously is a key to differentiating the sequential from parallel models, it is necessary to examine

\footnotetext{
${ }^{1}$ Wei et al. (2013) investigated how word properties of a currently fixated word could affect the following forward saccade length in two experiments. In their second experiment, the critical region was either a high-frequency word or a low-frequency word. They found the outgoing saccade length was longer in the high-frequency condition than in the low-frequency condition.
} 
whether there are parafoveal-on-foveal effects of word frequency in Chinese reading. Because single-character word frequency has a high correlation with character frequency, we cannot tease apart the effects of character or word frequency using single-character words. In the current study, we used two-character words as target words, and we manipulated character frequency and word frequency independently to examine whether there were parafoveal-on-foveal effects of character frequency or word frequency. Note that in normal Chinese reading, there are no spaces between words and each character occupies a relatively small degree of visual angle $\left(0.7^{\circ}\right.$ in this study), thus nonfixated characters or words sometimes appear in the fovea rather than in the parafovea. Therefore, in this study, we use the term "parafoveal-on-foveal effect" to refer to findings whereby the reading time on a word is affected by the frequency of the nonfixated successor word or character(s) in the nonfixated successor word.

In Experiment 1A, each pair of the two-character target words had a similar word frequency and shared the same second character while the first character was either a high- or lowfrequency character. We embedded each pair of words into the same sentence frame to examine whether the frequency of the first character of the target word affected fixation durations on the pre-target word. In Experiment 1B, each pair of two-character target words also shared the same second character, but the frequency of the word as a whole was either high or low. The first character of each pair of words was different but had similar character frequency. We embedded each pair of words in the same sentence frame to examine whether the frequency of the target word affected fixation duration on the pre-target word. We did not manipulate the character frequency and word frequency in a single sentence frame. Different subjects participated in Experiment 1A and 1B. To make the two conditions more comparable, we performed Experiment 2 using a within-subjects design. Each participant read the sentences from both Experiments 1A and 1B.

Parallel and sequential models of eye movement control differed in their predictions for outcomes of this study. The parallel models assume that multiple words are processed simultaneously, and thus, predicted a parafoveal-on-foveal effect of word frequency (Engbert et al., 2005; Risse, Engbert, \& Kliegl, 2008). In contrast, the sequential models assume that words are processed serially, and therefore, predicted no such effect of word frequency (Reichle, Warren, \& McConnell, 2009). At the character level, these two types of models do not make any assumptions because both types assumed words to be the basic unit of attention allocation and cognitive processing (see also the Chinese version of the E-Z Reader model, Rayner, Li, \& Pollatsek, 2007). However, a model on Chinese word segmentation and recognition has assumptions at both the character and word levels that should be noted ( $\mathrm{Li}$ et al., 2009). In Li et al.'s model, characters in the perceptual span can be processed in parallel (restricted by visual acuity) (see also M. Yan, Zhou, Shu, \& Kliegl, 2015), but words are processed in a serial order. Based on this model, character frequency instead of word frequency should affect fixation durations on pre-target words. 


\section{Experiment 1A and 1B}

\section{Method}

Participants-Forty-eight undergraduates from the China Agricultural University were paid to participate in this experiment. Half of the subjects participated in Experiment $1 \mathrm{~A}$ and half in Experiment 1B.

Apparatus-The materials were presented on a 21-inch CRT monitor (Sony G520: resolution: $1024 \times 768$ pixels; refresh rate: $150 \mathrm{~Hz}$ ) connected to a Dell PC. Each sentence was displayed on a single line in Song 20-point font, and the characters were shown in white (RGB: 256, 256, 256) on a black background (RGB: 0, 0, 0). Participants were seated at a viewing distance of $58 \mathrm{~cm}$ from the computer monitor. At this viewing distance, each character subtended a visual angle of approximate $0.7^{\circ}$. The head was stabilized with a chin rest and a forehead rest. Participants read sentences binocularly, but only the right eye was monitored. Eye movements were recorded by an EyeLink 1000 eye tracking system with a sampling rate of $1000 \mathrm{~Hz}$.

Materials and design-Forty-eight pairs of two-character words were selected as target words for Experiments 1A and another forty-eight pairs of two-character words were selected for Experiment 1B. In Experiment 1A, the pairs of two-character words had either a high- or low-frequency first character but an identical second character. Based on a published lexicon database (Chinese Linguistic Data Consortium, 2003), we calculated character and word frequency using occurrences per million words as a standardized measure $^{2}$. The frequency of the first character in the high-frequency character condition (HF-C) $(M=3,076$ occurrences per million words, $S D=2120)$ was greater than that for the low-frequency character condition (LF-C) $(M=235$ occurrences per million words, $S D=$ $156), t(47)=9.54, p<.001$. There was no difference in word frequency between the HF-C $(M=2.4$ occurrences per million words, $S D=3.4)$ and LF-C conditions $(M=2.4$ occurrences per million words, $S D=3.3), t<1$. The numbers of strokes in the first character did not differ between the HF-C condition $(M=7.2, S D=2.4)$ and the LF-C condition $(M=$ 7.3, $S D=2.4), t<1$, nor did the number of radicals in the HF-C $(M=2.1, S D=0.9)$ differ from the LF-C $(M=2.0, S D=0.7), t<1$, either.

In Experiment 1B, forty-eight pairs of two-character words with identical second characters within each pair were selected. For each pair of words, one word was high frequency and the other was low frequency. Word frequency in the high-frequency word condition (HF-W) (M $=33.4$ occurrences per million words, $S D=12.8$ ) was higher than that for the low-frequency word condition $(\mathrm{LF}-\mathrm{W})(M=0.3$ occurrences per million words, $S D=0.2), t(47)=17.94, p$ $<.001$. There was no difference in the frequency of the first character between the HF-W ( $M$ $=592$ occurrences per million words, $S D=521)$ and LF-W conditions $(M=539$ occurrences per million words, $S D=647), t<1$. The number of strokes in the first character was also

\footnotetext{
${ }^{2}$ When using Zipf-scale (log10(frequency per million words)+3) to standardize word frequency (van Heuven, Mandera, Keuleers, \& Brysbaert, 2014), word frequency in the HF-C condition $(M=3.1, S D=.55)$ in Experiment 1A was marginally smaller than that in the LF-C condition $(M=3.2, S D=.50), t=-1.95, p=.057$. Word frequency in the HF-W condition $(M=4.5, S D=.15)$ in Experiment 1B was significantly larger than that in the LF-W condition $(M=2.3, S D=.36), t=36.62, p<.001$.
} 
matched for the HF-W $(M=8.2, S D=2.0)$ and LF-W conditions $(M=8.1, S D=2.1), t<1$. The number of radicals in the HF-W condition $(M=2.1, S D=0.5)$ did not differ from the LF-W condition $(M=2.1, S D=0.5), t<1$, either.

Each pair of two-character words was embedded into the same sentence frame (see Figure 1). Forty-eight pairs of sentences were created for Experiments 1A and 1B, respectively, and all of the 96 sentence frames were different. The length of the sentences ranged from 20 to 25 characters. The words immediately before the target words in all of the trials were twocharacter words. All of the sentences used in Experiment 1A and 1B were selected from the same online corpus developed by Center for Chinese Linguistics, $\mathrm{PKU}^{3}$. The predictability of the target word was close to zero, as assessed by ten additional participants who did not participate in the formal experiments. We also asked twenty-four volunteers to assess the plausibility of target words in each condition on a 5-point scale $(1=$ very implausible; $5=$ very plausible; see Yang, Staub, Li, Wang, \& Rayner, 2012 for a similar method). The average plausibility scores did not significantly differ between the HF-C $(M=3.3, S D=0.9)$ and LF-C conditions $(M=3.4, S D=0.9), t \mathrm{~s}<1$. No significant differences were found between the HF-C $(M=3.0, S D=0.8)$ and LF-C conditions $(M=3.1, S D=0.9), t \mathrm{~s}<1.38$, $p$ s > .173, either. The twenty-four volunteers also assessed the ease of sentence comprehension on a 5 -point scale $(1=$ very difficult $; 5=$ very easy $)$ among each condition. The data revealed all the HF-C $(M=4.3, S D=0.4)$, LF-C $(M=4.3, S D=0.4)$, HF-W $(M=$ $4.1, S D=0.5)$, and LF-W $(M=4.2, S D=0.4)$ conditions were equally easy to understand, $t \mathrm{~s}$ $<1$.

Procedure-When participants came into the laboratory, they were given instructions for the experiment and a brief description of the apparatus. The chair was then adjusted to make the participants feel comfortable. The eye tracker was calibrated at the beginning of the experiment and was calibrated again as needed (recalibration was conducted after about every ten trials or a drift check failure). A three-point calibration and validation procedure was used. The maximum error of validation was 0.5 degrees in visual angle. Next, each participant read 6 sentences for practice and 72 sentences ( 24 fillers) for the formal experiment in a random order. The participants were asked to read silently and answer questions following one third of the sentences. The questions were created to make sure participants read the sentences carefully. Each sentence appeared after participants successfully fixated on a character-sized box at the location of the first character of each sentence. After reading a sentence, the participants were asked to press a response button to start the next trial.

Data analysis-Accuracy on the comprehension questions was high (95\%), suggesting that the participants understood the sentences very well. Trials in which participants blinked more than three times, or blinked once when they fixated on the target word, were excluded from the analysis, resulting in a loss of $3 \%$ of the trials. Fixations with durations longer than $800 \mathrm{~ms}$ or shorter than $80 \mathrm{~ms}$ (approximately $2 \%$ of all fixations) were also excluded from the analysis.

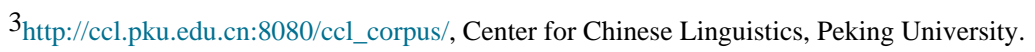


We mainly analyzed first-fixation duration (the duration of the first fixation on the target region, irrespective of the number of fixations) and gaze duration ${ }^{4}$ (the sum of all fixation durations on the target region before moving to another region) on both the target words and pre-target words (Rayner, 1998, 2009). The data were analyzed using a linear mixed-effects model (LMM) for continuous variables and a generalized mixed-effect model for binary variables (Baayen, Davidson, \& Bates, 2008; Jaeger, 2008), including maximal random effect structures as suggested by Barr, Levy, Scheepers, \& Tily (2013). Notice that fixation durations were log-transformed to better meet LMM assumptions (Kliegl, Masson, \& Richter, 2010). The Lme4 package (version 0.999999-2, Bates, Maechler, \& Bolker, 2013) was used for data analysis in the R environment (R Core Team, 2013). Given a relatively large sample set for LMM analysis, and because the t-distribution approximates a normal distribution, t-values greater than 1.96 were considered significant at the 5\% level (see also M. Yan \& Sommer, 2015).

We also analyzed the distribution of the fixation durations if we found a significant parafoveal-on-foveal effect at any measurement. This analysis could provide more information beyond the average fixation durations. The distribution of fixation durations can be used to examine whether this effect is caused by mislocated fixations (see details in the discussion section). A non-parametric method of vincentile plotting (Ratcliff, 1979) was used to visualize the distribution. For each participant in each condition, fixation durations were ranked and divided into 10 bins. The first bin contained the shortest 10\% (vincentile 1) of the data; the second bin contained the next shortest $10 \%$ (vincentile 2), and so on. Then, the mean of each participant in each condition was computed for each vincentile. Finally, we plotted the vincentile points and performed t-tests to determine which bins differed significantly between the two conditions.

Furthermore, we fit the distribution to an ex-Gaussian function (Staub, White, Drieghe, Hollway, \& Rayner, 2010; White \& Staub, 2012) to investigate how the distributions of the two conditions differed. The ex-Gaussian function is a combination of a Gaussian normal distribution and an exponential distribution specified by three parameters: $\mu$ (the mean of the distribution), $\sigma$ (the variability of the distribution), and $\tau$ (the degree of rightward skew of the distribution). Using this method, we could further explore the extent to which the average differences reflected an effect on most of the fixations or only a subset of them. First-fixation durations on pre-target words were fit for each participant in each condition using the timefit function in the retimes package (Massidda, 2013). The timefit function uses maximum likelihood estimation to determine the three parameters $(\mu, \sigma$, and $\tau)$ with 20,000 random samples. The estimated parameters for each participant in each condition were analyzed using t-tests to determine which parameters differed between the two conditions. A significant difference of $\mu$ between the two conditions would indicate that readers extracted

\footnotetext{
${ }^{4}$ On pre-target word regions, the other indicator such as go-past time, revealed the same trend as gaze duration. Go-past times were shorter in the HF-C condition ( $M=322 \mathrm{~ms}, S D=47 \mathrm{~ms})$ than in the LF-C condition $(M=352 \mathrm{~ms}, S D=50 \mathrm{~ms})$ in Experiment $1 \mathrm{~A}, b=$ $-0.035, S E=0.011, t=-3.28$, but go-past times did not differ between the HF-C ( $M=323 \mathrm{~ms}, S D=61 \mathrm{~ms})$ and LF-C conditions $(M$ $=328 \mathrm{~ms}, S D=60 \mathrm{~ms}$ ) in Experiment $2 \mathrm{~A}, t<1$. In addition, word frequency did not show parafoveal-on-foveal effect in go-past time, either. There were no significant differences in go-past time for Experiment $1 \mathrm{~B}$ between the HF-W $(M=356 \mathrm{~ms}, S D=60 \mathrm{~ms})$ and LFW conditions $(M=351 \mathrm{~ms}, S D=62 \mathrm{~ms}), t<1$, or for Experiment 2B between the HF-W $(M=330 \mathrm{~ms}, S D=67 \mathrm{~ms})$ and LF-W conditions $(M=326 \mathrm{~ms}, S D=69 \mathrm{~ms}), t<1$.
} 
parafoveal frequency information in most fixations. In contrast, if there was only a significant difference of $\tau$ between the two conditions, then this would mean that the average differences were caused by a rightward skew of the distribution and that readers only extracted parafoveal frequency information in long fixations.

\section{Results and discussion}

Pre-target word region-In Experiment 1A, the frequency of the first character of the two-character target word affected fixation durations on the pre-target words. First-fixation durations on pre-target words were shorter in the HF-C condition ( $M=266 \mathrm{~ms}, S D=24 \mathrm{~ms})$ than in the LF-C condition ( $M=282 \mathrm{~ms}, S D=27 \mathrm{~ms}), b=-0.021, S E=0.009, t=-2.18$. Gaze durations on the pre-target words were numerically shorter in the HF-C condition ( $M=$ $303 \mathrm{~ms}, S D=45 \mathrm{~ms})$ than in the LF-C condition $(M=327 \mathrm{~ms}, S D=45 \mathrm{~ms}), b=-0.027, S E$ $=0.014, t=-1.92$. We further found that these parafoveal-on-foveal effects were attributed to long pre-target fixations (see Figure 2 and Figure 4). Upon fitting first-fixation durations on pre-target words with an ex-Gaussian function, we found that $\tau$ was significantly smaller in the HF-C condition $(M=65, S D=32)$ than in the LF-C condition $(M=87, S D=31)$, $t(23)=-2.36, p=.026$. In contrast, there were no differences for parameter $\mu$ (HF-C: $M=$ 199, $S D=40$, LF-C: $M=195, S D=43$ ), $t<1$, or $\sigma$ (HF-C: $M=37, S D=22$, LF-C: $M=34$, $S D=17), t<1$. Vincentile-plotting visually confirmed that first-fixation durations on pretarget words were shorter in the HF-C condition than the LF-C condition only for vincentiles $7-9, p s<.05$.

To investigate whether visual limitation may constrain the parafoveal-on-foveal effects, we divided trials into two groups according to landing positions on the pre-target word (Inhoff, Radach, Starr, \& Greenberg, 2000; Zhou, Kliegl, \& Yan, 2013). In one group of trials (57\% of trials), first-fixations were located on the first characters of the 2-character pre-target words, and in the other group ( $43 \%$ of trials), the fixations were located on the second characters. When the second characters of pre-target words were fixated, first-fixation durations on the pre-target words were $22 \mathrm{~ms}$ shorter in the HF-C condition $(M=272 \mathrm{~ms}$, $S D=35 \mathrm{~ms}$ ) than in the LF-C condition ( $M=294 \mathrm{~ms}, S D=42 \mathrm{~ms}), b=-0.028, S E=0.015$, $t=-1.96$. In contrast, when the first characters of pre-target words were fixated, firstfixation durations on the pre-target words were numerically $13 \mathrm{~ms}$ shorter in the HF-C condition $(M=262 \mathrm{~ms}, S D=29 \mathrm{~ms})$ than in the LF-C condition $(M=275 \mathrm{~ms}, S D=36 \mathrm{~ms})$, but the difference was not significantly different, $b=-0.017, S E=0.014, t=-1.25$. These results suggest that it is possible that visual limitation constrained parafoveal-on-foveal effects in our sample.

In Experiment 1B, a parafoveal-on-foveal effect of word frequency was not observed (see also White, 2008 in English). There were no statistically significant differences between the HF-W and LF-W conditions in either first-fixation duration (HF-W: $M=292 \mathrm{~ms}, S D=36$ ms; LF-W: $M=291 \mathrm{~ms}, S D=40 \mathrm{~ms}$ ), $t<1$, or gaze duration (HF-W: $M=336 \mathrm{~ms}, S D=58$ ms; LF-W: $M=334 \mathrm{~ms}, S D=68 \mathrm{~ms}$ ), $t<1$. As in Experiment 1A, we divided first-fixation durations into two groups according to the landing positions on the pre-target word. There were still no statistically significant differences between the HF-W and LF-W conditions in either the first fixations located on the first characters of pre-target words (HF-W: $M=286$ 
$\mathrm{ms}, S D=39 \mathrm{~ms}$; LF-W: $M=287 \mathrm{~ms}, S D=44 \mathrm{~ms}), t<1$, or located on the second characters (HF-W: $M=303 \mathrm{~ms}, S D=50 \mathrm{~ms}$; LF-W: $M=292 \mathrm{~ms}, S D=48 \mathrm{~ms}$ ), $b=0.017, S E=0.013$, $t=1.27$. We used ttestBF function in the package BayesFactor (Morey \& Rouder, 2013; Rouder, Morey, Speckman, \& Province, 2012; Rouder, Speckman, Sun, Morey, \& Iverson, 2009) in the R environment (R Core Team, 2013) to test the reliability of the lack of effect of word frequency. Our results revealed that the null hypotheses of no parafoveal-on-foveal effect were 5.3 and 5.1 times more likely to be true than the alternative hypotheses for firstfixation duration and gaze duration, respectively. Therefore, the results of the current study are consistent with the argument that Chinese readers are more likely to extract character information in the parafovea. However, we did not find evidence that Chinese readers could extract information on two-character words before fixating on it within the current design of the experiment.

Target word region-In Experiment 1A, there were no significant differences between the HF-C and LF-C conditions in either first-fixation duration on the target words (HF-C: $M$ $=305 \mathrm{~ms}, S D=30 \mathrm{~ms}$; LF-C: $M=298 \mathrm{~ms}, S D=34 \mathrm{~ms}$ ), $t<1$, or gaze duration (HF-C: $M=$ $361 \mathrm{~ms}, S D=42 \mathrm{~ms}$; LF-C: $M=350 \mathrm{~ms}, S D=40 \mathrm{~ms}$ ), $t<1$. The absence of an effect from character frequency on whole word recognition time is consistent with a corpus analysis by Li et al. (2014). In Experiment 1B, we found a reliable word frequency effect on the target region. First-fixation durations were shorter in the HF-W condition $(M=281 \mathrm{~ms}, S D=25$ $\mathrm{ms}$ ) than in the LF-W condition $(M=303 \mathrm{~ms}, S D=39 \mathrm{~ms}), b=-0.025, S E=0.011, t=$ -2.23 . Gaze durations on target words were also shorter in the HF-W condition $(M=333$ $\mathrm{ms}, S D=48 \mathrm{~ms})$ than in the LF-W condition $(M=371 \mathrm{~ms}, S D=59 \mathrm{~ms}), b=-0.039, S E=$ $0.013, t=-2.86$. The word frequency effect is consistent with several previous studies on Chinese reading (Li et al., 2014; Wei et al., 2013; G. Yan, Tian, Bai, \& Rayner, 2006).

In Experiment 1A, fixation probability on the target word ${ }^{5}$ was $.89(S D=.11)$ in the HF-C condition, and was similar to that in the LF-C condition $(\mathrm{M}=.89, S D=.12)$. Since whether a word is fixated affects the duration of the fixations on the previous word (Kliegl \& Engbert, 2005), we analyzed fixations on the pre-target word separately depending on whether the target word was fixated or skipped. When the target word was fixated, firstfixation durations on pre-target words were significantly shorter in the HF-C condition $(M=$ $269 \mathrm{~ms}, S D=25 \mathrm{~ms})$ than in the LF-C condition $(M=280 \mathrm{~ms}, S D=30 \mathrm{~ms}), b=-0.021, S E$ $=0.010, t=-1.99$. When the target word was skipped, first-fixation durations on pre-target words were numerically, but not statistically shorter in the HF-C condition $(M=264 \mathrm{~ms}, S D$ $=40 \mathrm{~ms})$ than in the LF-C condition $(M=283 \mathrm{~ms}, S D=51 \mathrm{~ms}), b=-0.031, S E=0.024, t=$

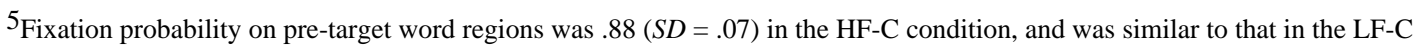
condition $(M=.86, S D=.09)$ in Experiment 1A. First-fixation durations on target words were not significantly different between the HF-C and LF-C condition, no matter whether the pre-target words were skipped (HF-C: $M=305 \mathrm{~ms}, S D=65 \mathrm{~ms}$; LF-C: $M=303 \mathrm{~ms}$, $S D=87 \mathrm{~ms}$ ) or fixated (HF-C: $M=295 \mathrm{~ms}, S D=34 \mathrm{~ms}$; LF-C: $M=306 \mathrm{~ms}, S D=34 \mathrm{~ms}$ ), $t \mathrm{~s}<1$. In Experiment $1 \mathrm{~B}$, fixation probability on pre-target word regions was $.88(S D=.13)$ in the HF-W condition, and was similar to that in the LF-W condition $(M=$. $89, S D=.10$ ). When the target word was fixated, first-fixation durations on pre-target words were significantly shorter in the HF-W condition $(M=286 \mathrm{~ms}, S D=26 \mathrm{~ms})$ than in the LF-W condition $(M=311 \mathrm{~ms}, S D=40 \mathrm{~ms}), \mathrm{b}=-0.028, \mathrm{SE}=0.009, t=-2.98$. When the target word was skipped, first-fixation durations on pre-target words were numerically, but not statistically shorter in the HF-W condition ( $M=258 \mathrm{~ms}, S D=48 \mathrm{~ms})$ than in the LF-W condition $(M=267 \mathrm{~ms}, S D=73 \mathrm{~ms}), t<1$. The null effect might be because only about $10 \%$ of trials were included when target words were skipped. Similar results were observed in Experiment 2 . Notice that this study was designed to investigate whether character or word frequency of nonfixated-target words could affect the fixation durations on currently fixated-pretarget words, thus we would not discuss too much on eye movement measures on target words.
} 
-1.29 . The null effect might be because only about $10 \%$ of trials were included when target words were skipped. In Experiment 1B, fixation probability was .91 $(S D=.10)$ for the HF$\mathrm{W}$ condition and was $.90(S D=.11)$ for the LF-W condition. When the target word was fixated, first-fixation durations were not significantly different between the HF-W ( $M=297$ $\mathrm{ms}, S D=36 \mathrm{~ms}$ ) and LF-W conditions $(M=297 \mathrm{~ms}, S D=47 \mathrm{~ms}), t<1$; When the target word was skipped, first-fixation durations were still not significant different between the HF-W $(M=274 \mathrm{~ms}, S D=118 \mathrm{~ms})$ and LF-W conditions $(M=266 \mathrm{~ms}, S D=69 \mathrm{~ms}), t<1$.

\section{Experiment 2}

In Experiment 1A, we observed reliable parafoveal-on-foveal effects of character frequency, but in Experiment 1B, we did not find parafoveal-on-foveal effects of word frequency. Because different participants participated in Experiments $1 \mathrm{~A}$ and 1B, it is possible that the phenomenon observed in Experiment 1A and 1B were caused by differences in participants. To exclude this possibility, we carried out Experiment 2. In contrast to the between-subjects design employed for the character and word frequency manipulations in Experiment $1 \mathrm{~A}$ and 1B, Experiment 2 involved a within-subjects design for these two variables. A new group of 30 participants from the same participant pool participated in Experiment 2. For convenience of comparison, the character frequency manipulation experiment is referred to as Experiment 2A and the word frequency manipulation experiment as Experiment 2B. All of the procedures, stimuli and analysis methods were identical to those described for Experiments 1A and 1B. The average comprehension accuracy was $94 \%$, which suggested that the readers comprehended all of the sentences very well. Approximately $6 \%$ of the trials were excluded because of mismatching of the same selection criterion used in Experiment $1 \mathrm{~A}$ and $1 \mathrm{~B}$.

\section{Results and discussion}

Pre-target word region-As was expected, the interaction between character and word frequency manipulations were statistically significant, $b=-0.025, S E=0.011, t=-2.25$. The main result of Experiment 1A was reproduced in Experiment 2A. Character frequency significantly modulated fixation durations on the pre-target words. First-fixation durations on pre-target words were shorter in the HF-C condition ( $M=247 \mathrm{~ms}, S D=34 \mathrm{~ms}$ ) than in the LF-C condition ( $M=261 \mathrm{~ms}, S D=34 \mathrm{~ms}$ ), $b=-0.018, S E=0.008, t=-2.29$. Gaze durations ${ }^{6}$ on pre-target words did not significantly differ between the HF-C ( $M=290 \mathrm{~ms}$, $S D=54 \mathrm{~ms})$ and LF-C conditions $(M=298 \mathrm{~ms}, S D=54 \mathrm{~ms}), t<1$. Further analysis revealed that the parafoveal-on-foveal effects on first-fixation durations were also attributable to long pre-target fixations as in Experiment 1A (see Figure 3 and Figure 4). When we fit the fixation durations with the ex-Gaussian distribution, we found that parameter $\tau$ had a marginally significant lower value in the HF-C condition $(M=67, S D=$ $34)$ than in the LF-C condition $(M=82, S D=33), t(29)=-1.85, p=.074$. There were no differences in parameter $\mu$ (HF-C: $M=180, S D=38$, LF-C: $M=179, S D=31$ ), $t<1$, or $\sigma$ (HF-C: $M=30, S D=18$, LF-C: $M=33, S D=20$ ), $t<1$. Vincentile-plotting confirmed this

\footnotetext{
${ }^{6}$ The lack of a parafoveal-on-foveal effect of character frequency on gaze duration might be caused by type I error, but the parafovealon-foveal effect of character frequency was replicated for first-fixation duration. Moreover, the effect was attributed to long pre-target fixations in Experiment 2A, as well as in Experiment 1A.
} 
finding and revealed a steep slope on the right side. The t-tests demonstrated that, as in Experiment 1A, only vincentiles 7-9 showed significant differences between the HF-C and LF-C conditions, $p s<.05$.

We divided trials into two groups according to landing positions (58\% and $42 \%$ of trials located on the first and second character, respectively) as we did in Experiment $1 \mathrm{~A}$. The results showed that first-fixation durations on the pre-target words were $18 \mathrm{~ms}$ shorter in the HF-C condition $(M=235 \mathrm{~ms}, S D=33 \mathrm{~ms})$ than in the LF-C condition $(M=253 \mathrm{~ms}, S D=$ $33 \mathrm{~ms}), b=-0.026, S E=0.011, t=-2.37$, when the first characters of pre-target words were fixated. In contrast, when the second characters of pre-target words were fixated, firstfixation durations did not significantly differ between the HF-C $(M=250 \mathrm{~ms}, S D=45 \mathrm{~ms})$ and LF-C conditions $(M=249 \mathrm{~ms}, S D=39 \mathrm{~ms}), t<1$. The influences of fixation position on the size of parafoveal-on-foveal effects were different between this experiment and Experiment 1A. In Experiment 1A, larger parafoveal-on-foveal effects were observed when readers fixated much more closely to the target words. However, in Experiment 2A, the parafoveal-on-foveal effect of character frequency was bigger when readers fixated further from the target words, and the effect disappeared altogether when readers fixated closer to the target word. The finding of a different pattern of results regarding the relation between landing positions on pre-target words and the size of parafoveal-on-foveal effects is not unique to the current study. Previous studies have also found that the relationship between landing positions on the pre-target words and the size of parafoveal-on-foveal effects might not be strictly linear as reported in a corpus analysis by Kennedy (2008), where they found that parafoveal-on-foveal effects could occur at fixations closer (potentially mislocated) or further (less likely mislocated) to target words. In addition, Zhou et al. (2013) also reported that the semantic preview benefit was smaller when readers fixated closer to the target words. Given the mixed findings regarding the relationship between visual limitation and parafoveal information extraction, we will not discuss these results further.

In Experiment 2B, the lack of parafoveal-on-foveal effects of word frequency was reproduced. There was no statistically significant difference between the HF-W and LF-W conditions in either first-fixation duration (HF-W: $M=267 \mathrm{~ms}, S D=42 \mathrm{~ms}$; LF-W: $M=261$ $\mathrm{ms}, S D=32 \mathrm{~ms}$ ), $t<1$, or gaze duration (HF-W: $M=307 \mathrm{~ms}, S D=59 \mathrm{~ms}$; LF-W: $M=300$ $\mathrm{ms}, S D=51 \mathrm{~ms}), t<1$. We further analyzed first-fixation durations according to landing positions. The results did not reveal significant differences between the HF-W and LF-W conditions in either the first fixations located on the first characters of pre-target words (HF$\mathrm{W}: M=260 \mathrm{~ms}, S D=47 \mathrm{~ms}$; LF-W: $M=248 \mathrm{~ms}, S D=30 \mathrm{~ms}), t<1$, or the first fixations located on the second characters (HF-W: $M=261 \mathrm{~ms}, S D=45 \mathrm{~ms}$; LF-W: $M=264 \mathrm{~ms}, S D$ $=40 \mathrm{~ms}), t<1$. Consistent with Experiment 1B, the BayesFactor analysis revealed that the null hypotheses of no parafoveal-on-foveal effects were 5.4 and 5.6 times more likely to be true than the alternative hypotheses for first-fixation duration and gaze duration, respectively.

Target word region-The main results of the target region analyses in Experiments $1 \mathrm{~A}$ and $1 \mathrm{~B}$ were reproduced in Experiments $2 \mathrm{~A}$ and $2 \mathrm{~B}$. Character frequency manipulations did not affect fixation durations on the two-character words as a whole. There were no significant differences between the HF-C and LF-C conditions in either first-fixation 
duration (HF-C: $M=273 \mathrm{~ms}, S D=48 \mathrm{~ms}$; LF-C: $M=272 \mathrm{~ms}, S D=36 \mathrm{~ms}$ ), $t<1$, or gaze duration (HF-C: $M=326 \mathrm{~ms}, S D=70 \mathrm{~ms}$; LF-C: $M=314 \mathrm{~ms}, S D=60 \mathrm{~ms}$ ), $t<1$.

Furthermore, the word frequency effect was reproduced. First-fixation durations on target words were shorter in the HF-W condition $(M=267 \mathrm{~ms}, S D=38 \mathrm{~ms})$ than in the LF-W condition $(M=283 \mathrm{~ms}, S D=35 \mathrm{~ms}), b=-0.021, S E=0.011, t=-2.04$. Gaze durations on target words were also shorter in the HF-W condition $(M=303 \mathrm{~ms}, S D=45 \mathrm{~ms})$ than in the LF-W condition $(M=335 \mathrm{~ms}, S D=50 \mathrm{~ms}), b=-0.037, S E=0.011, t=-3.31$.

Similar to Experiment 1A and 1B, we analyzed fixation durations separately depending on whether the target words were fixated. In Experiment $2 \mathrm{~A}$, fixation probability on target word was $.83(S D=.12)$ in the HF-C condition, and was .82 $(S D=.11)$ in the LF-C condition, and the differences between these two conditions were not significant, $t<1$. When the target word was fixated, first-fixation durations on pre-target words were shorter in the HF-C condition $(M=247 \mathrm{~ms}, S D=36 \mathrm{~ms})$ than in the LF-C condition $(M=257 \mathrm{~ms}, S D=35 \mathrm{~ms})$, $b=-0.018, S E=0.008, t=-2.12$. When the target word was skipped, first-fixation durations did not significantly differ between the HF-C ( $M=245 \mathrm{~ms}, S D=73 \mathrm{~ms})$ and LF-C conditions ( $M=248 \mathrm{~ms}, S D=55 \mathrm{~ms}), t<1$. However, as in Experiment $1 \mathrm{~A}$ only about $18 \%$ of trials were included when the target word was skipped. In Experiment $2 \mathrm{~B}$, fixation probability was $.83(S D=.10)$ in the HF-W condition and was $.87(S D=.10)$ in the LF-W condition. The difference between these two conditions was significant, $b=-0.045, S E=$ $0.021, t=-2.07$. Similar to Experiment 1B, when the target word was fixated, first-fixation durations on pre-target words showed no significant differences between the HF-W ( $M=$ $268 \mathrm{~ms}, S D=43 \mathrm{~ms})$ and LF-W conditions $(M=257 \mathrm{~ms}, S D=33 \mathrm{~ms}), t<1$. When the target word was skipped, there was also no difference between the HF-W ( $M=258 \mathrm{~ms}, S D$ $=58 \mathrm{~ms})$ and LF-W conditions $(M=256 \mathrm{~ms}, S D=114 \mathrm{~ms}), t<1$.

\section{General discussion}

In this study, we examined whether there were parafoveal-on-foveal effects of character frequency or word frequency in Chinese reading. The frequency of the first character in a two-character target word (character frequency) was manipulated in Experiment 1A, and the frequency of the two-character target word (word frequency) was manipulated in Experiment 1B. We found that first-fixation durations and gaze durations on pre-target words were shorter when the first character of the two-character target word occurred with high rather than low frequency in Experiment 1A. Such parafoveal-on-foveal effects were not observed following word frequency manipulations in Experiment 1B. The parafovealon-foveal effect of character frequency was reproduced for first-fixation duration in Experiment 2A while the lack of parafoveal-on-foveal effect of word frequency was reproduced in Experiment 2B. These findings are important for understanding parafoveal processing and attention allocation in Chinese reading.

Previous studies have demonstrated parafoveal-on-foveal effects of one-character word frequency (M. Yan et al., 2010) and two-character word frequency (Bai et al., 2009) in Chinese reading, but it was unknown whether those effects occurred at the character level or word level. Because a single-character word is also a character and word frequency is highly correlated (approximately .95) with character frequency, the parafoveal-on-foveal effects of 
single-character word frequency potentially reflect the influence of character frequency (M. Yan et al., 2010). In another study, parafoveal-on-foveal effects of two-character word frequency were observed, but the frequency of the first character in the words was not matched (Bai et al., 2009). Therefore, the effects of two-character word frequency could potentially be due to character frequency. In this study, we matched these potential confounding factors to examine whether there were parafoveal-on-foveal effects of character frequency or word frequency. Consistent with the corpus analysis by Li et al. (2014), we found parafoveal-on-foveal effects of character frequency, but we did not observe such effects due to word frequency. These results suggest that parallel processing most likely occurred at the character level, rather than at the word level.

It could be argued that the lack of a parafoveal-on-foveal effect for word frequency may be because the frequency of the target words in the high-frequency condition was not high enough. We suspect that this was not the case for two main reasons. First, the target words in the high-frequency condition were frequent enough to produce word frequency effects on target words. Second, the frequency of the target words ranged between 18 and 77 occurrences per million. These words are among the top 5\% frequently used two-character words in the Chinese lexicon. Thus, we assume that the target words we used were representative high-frequency words, and therefore, the lack of a parafoveal-on-foveal effect for word frequency was unlikely caused by a limited word frequency range. However, we acknowledge that it might be interesting to replicate the study with extremely highfrequency words (words among the top $1 \%$ frequently used two-character words).

At the word level, the lack of parafoveal-on-foveal effects of word frequency is consistent with the predictions of sequential models such as the E-Z Reader model (Reichle et al., 1998, 2003, 2009). According to the E-Z Reader model, words are processed serially so that parafoveal word frequency does not influence fixation durations on the foveal word. In alphabetic languages, many experimental studies have demonstrated that parafoveal word frequency did not affect processing of the currently fixated word (Henderson \& Ferreira, 1993; White, 2008). In contrast, the lack of parafoveal-on-foveal effects of word frequency in this study is inconsistent with the predictions of the current state of parallel models. Parallel models assume that multiple words are processed in parallel (Engbert et al., 2005; Reilly \& Radach, 2006), and thus, predict parafoveal-on-foveal effects of word frequency. As indicated in the previous study (Kliegl et al., 2007), although saccade inhibition was only modulated by foveal processing load in the current state of the SWIFT model, in a general parallel processing view, saccade inhibition should be influenced by both the foveal and parafoveal processing loads. Therefore, an increase in parafoveal word difficulty (e.g., lowfrequency word) should delay saccade initiation (resulting in increased fixation durations). However, we did not find evidence for parallel processing of multiple words in the experiments.

It may be argued that the reason we did not find parafoveal-on-foveal effects may be because the perceptual span is not large enough to cover a whole two-character word. We argued that this should not be the major reason. In Chinese reading, the perceptual span includes one character to the left of current fixation and 2-3 characters to its right (Chen \& Tang, 1998; Inhoff \& Liu, 1998). When we divided first fixations into two groups according 
to landing positions, the fixations located on the second character were closer to target words. Thus, a whole two-character target word should fall within the effective perceptual span in this situation. Even so, we did not obtain any parafoveal-on-foveal effects for word frequency. These findings suggest that the lack of parafoveal-on-foveal effects of word frequency was unlikely caused solely by visual limitation.

At the character level, we observed stable parafoveal-on-foveal effects of character frequency in both Experiments 1A and 2A. Such effects could not be solely caused by mislocated fixations (Drieghe, Rayner, \& Pollatsek, 2008; Reichle \& Drieghe, 2015). Mislocated fixations can be attributed to the following three sources: imperfect binocular convergence of the eyes (Liversedge, White, Findlay, \& Rayner, 2006); oculomotor error associated with executing saccades (McConkie, Kerr, Reddix, \& Zola, 1988); and measurement error of the eye tracking technique (see Reichle \& Drieghe, 2015 for more information). When readers made a mislocated fixation on word $\mathrm{n}$, the actual attended and processed word would be word $n+1$. However, our data cannot be easily explained using the hypothesis of mislocated fixations. First, if the observed effects were caused solely by mislocated fixations, it would be expected the effects would have occurred for a wide range of fixations (both short and long fixations can be mislocated). However, the ex-Gaussian fitting and vincentile plotting revealed that the parafoveal-on-foveal effects of character frequency only occurred on long pre-target fixations. Second, when we separated first fixations into two groups by landing positions on pre-target words, we found that parafoveal-on-foveal effects were less likely to appear at mislocated fixations in Experiment $2 \mathrm{~A}$, although that was not consistently found in Experiment $1 \mathrm{~A}$. We can at least safely conclude that such effects cannot be solely explained by the hypothesis of mislocated fixations.

The parafoveal-on-foveal effect of character frequency is not easily explained by the current states of most popular eye movement control models for alphabetic languages. Because explicit spaces mark word boundaries in the alphabetic languages, both parallel and sequential models assume that a word is the basic unit of cognitive processing and attention allocation (Engbert et al., 2005; Reichle et al., 1998). Thus, it might be unnecessary to consider sublexical representations, such as morpheme processing, in a general reading model with western languages such as English. However, there are no spaces marking word boundaries in Chinese text. The character, instead of the word, is the most salient unit in Chinese text. Moreover, most characters are also single-character words and have their own meanings. These special properties ensure that characters play a critical role in Chinese reading, and therefore, it is not unexpected to observe parafoveal-on-foveal effects of character frequency.

The parafoveal-on-foveal effect of character frequency is consistent with Li et al.'s (2009) model, which assumed that characters are processed in parallel but words are processed serially. Although Li et al. (2009) assumed characters in the perceptual span could be processed in parallel, the processing rate might be constrained by visual eccentricity and word boundaries. In Li et al.'s study, readers were briefly presented with, and then reported, two 2-character words or one 4-character word. They found a larger drop in accuracy between the second and third character position in the two-word condition. $\mathrm{Li}$ and $\mathrm{Ma}$ 
(2012) used a probe detection task to further confirm the word boundary effect. Chinese readers saw four Chinese characters briefly, and then a probe was shown at one of the four character positions. The four characters constituted either one 4-character word or two 2character words. They found that reaction time was shorter in the second character position than in the third position in the two-word condition. Therefore, word boundaries might restrict parallel processing of multiple characters in the perceptual span. Only for long pretarget fixations could readers accumulate enough information about the parafoveal character of the next word. This is one possible reason why we observed parafoveal-on-foveal effects of character frequency for long pre-target fixations.

Compared with alphabetic languages, the structure of Chinese script might provide more possibilities for readers to extract morphological information (Yen, Tsai, Tzeng, \& Hung, 2008). The Chinese character is like a morpheme in English compound words, but the morpheme in English words is not as salient as the Chinese character in natural sentences. For example, in the sentence "I like playing football," foot is a morpheme which is tightly connected to the second morpheme ball. It might be difficult for readers to segment out the first morpheme using parafoveal vision. Therefore, it is not unusual that there is no reliable evidence for extracting the initial morpheme frequency in parafoveal vision in both English (Andrews, Miller, \& Rayner, 2004; Juhasz, 2008) and Finnish (Bertram \& Hyönä, 2003; Hyönä \& Bertram, 2004; Hyönä \& Pollatsek, 1998; Pollatsek \& Hyönä, 2005). In contrast, as illustrated in the Chinese translation “我喜欢踢足球” (I like playing football), each character is segmented with narrow spaces. Because there are no inter-word spaces marking word boundaries, the character instead of the word is the most salient unit in Chinese sentences. Therefore, it is more practical for readers to extract character instead of word information using parafoveal vision.

To summarize, we observed reliable parafoveal-on-foveal effects of character frequency, but not word frequency, in Chinese reading. These results are consistent with those claims that characters might be processed in parallel. However, in the current experiment we did not find evidence that multiple words are processed in parallel when reading Chinese.

\section{Supplementary Material}

Refer to Web version on PubMed Central for supplementary material.

\section{Acknowledgements}

We thank Marc Brysbaert, Ming Yan, Elizabeth Schotter and Albrecht Inhoff for their helpful comments on an earlier version of this work. This research was supported by the Knowledge Innovation Program of the Chinese Academy of Sciences (KSCX2-YW-BR-6), by a grant from the Natural Science Foundation of China (31070904), and by grant HD065829 from the US National Institute of Health.

\section{References}

Andrews S, Miller B, Rayner K. Eye movements and morphological segmentation of compound words: There is a mouse in mousetrap. European Journal of Cognitive Psychology. 2004; 16(1-2): 285-311. 
Baayen RH, Davidson DJ, Bates DM. Mixed-effects modeling with crossed random effects for subjects and items. Journal of Memory and Language. 2008; 59(4):390-412. doi: 10.1016/j.jml. 2007.12.005.

Bai X, Hu X, Yan G. An eye movement study on the role of nonfixated word characteristics in the fixated word's processing. Journal of Psychological Science (China). 2009; 32(2):308-311.

Barr DJ, Levy R, Scheepers C, Tily HJ. Random effects structure for confirmatory hypothesis testing: Keep it maximal. Journal of Memory and Language. 2013; 68(3):255-278. doi: 10.1016/j.jml. 2012.11.001.

Bates, D.; Maechler, M.; Bolker, B. lme4: Linear mixed-effects models using S4 classes (R package version 0.999999-2). 2013. Retrieved from http://CRAN.R-project.org/package=lme4

Bertram R, Hyönä J. The length of a complex word modifies the role of morphological structure: Evidence from eye movements when reading short and long Finnish compounds. Journal of Memory and Language. 2003; 48(3):615-634. doi: 10.1016/s0749-596x(02)00539-9.

Chen HC, Tang CK. The effective visual field in reading Chinese. Reading and Writing: An Interdisciplinary Journal. 1998; 10:245-254.

Chinese Linguistic Data Consortium. 现代汉语通用词表 [Chinese lexicon] (CLDC-LAC-2003-001). Tsinghua University, State Key Laboratory of Intelligent Technology and Systems, and Chinese Academy of Sciences, Institute of Automation; Beijing, China: 2003.

Cui L, Wang S, Yan G, Bai X. Parafoveal-on-foveal interactions in normal Chinese reading. Acta Psychologica Sinica (China). 2010; 42(2):547-558.

Cui L, Yan G, Bai X, Hyönä J, Wang S, Liversedge SP. Processing of compound-word characters in reading Chinese: An eye-movement-contingent display change study. The Quarterly Journal of Experimental Psychology. 2013; 66(3):527-547. [PubMed: 22809368]

Drieghe D, Rayner K, Pollatsek A. Mislocated fixations can acount for parafoveal-on-foveal effects in eye movements during reading. The Quarterly Journal of Experimental Psychology. 2008; 61(8): 1239-1249. [PubMed: 17853202]

Engbert, R.; Kliegl, R. Parallel graded attention models of reading.. In: Liversedge, SP.; Gilchrist, ID.; Everling, S., editors. The Oxford handbook of eye movements. Oxford University Press; Oxford, England: 2011. p. 787-800.

Engbert R, Nuthmann A, Richter EM, Kliegl R. SWIFT: A dynamical model of saccade generation during reading. Psychological Review. 2005; 112(4):777-813. doi: 10.1037/0033-295x.112.4.777. [PubMed: 16262468]

Henderson JM, Ferreira F. Eye movement control during reading: Fixation measures reflect foveal but not parafoveal processing difficulty. Canadian Journal of Experimental Psychology. 1993; 47(2): 201. [PubMed: 8364530]

Hoosain, R. Psycholinguistic implications for linguistic relativity: A case study of Chinese. Lawrence Erlbaum Associates, Inc; Hillsdale, NJ: 1991.

Hyönä J, Bertram R. Do frequency characteristics of nonfixated words influence the processing of fixated words during reading? European Journal of Cognitive Psychology. 2004; 16(1-2):104-127.

Hyönä J, Pollatsek A. Reading Finnish compound words: Eye fixations are affected by component morphemes. Journal of Experimental Psychology: Human Perception and Performance. 1998; 24(6):1612-1627. [PubMed: 9861713]

Inhoff AW, Liu WM. The perceptual span and oculomotor activity during the reading of Chinese sentences. Journal of Experimental Psychology: Human Perception and Performance. 1998; 24(1): 20-34. [PubMed: 9483822]

Inhoff, AW.; Radach, R.; Starr, M.; Greenberg, S. Allocation of visuo-spatial attention and saccade programming during reading.. In: Kennedy, A.; Radach, R.; Heller, D.; Pynte, J., editors. Reading as a perceptual process. Elsevier; Amsterdam: 2000. p. 221-246.

Inhoff AW, Starr M, Shindler KL. Is the processing of words during eye fixations in reading strictly serial? Perception \& Psychophysics. 2000; 62(7):1474-1484. [PubMed: 11143457]

Jaeger TF. Categorical data analysis: Away from ANOVAs (transformation or not) and towards logit mixed models. Journal of Memory and Language. 2008; 59(4):434-446. [PubMed: 19884961] 
Juhasz BJ. The processing of compound words in English: Effects of word length on eye movements during reading. Language and Cognitive Processes. 2008; 23(7-8):1057-1088. doi: 10.1080/01690960802144434.

Kennedy, A. The influence of parafoveal words on foveal inspection time: Evidence for a processing trade-off.. In: Underwood, G., editor. Eye guidance in reading and scene perception. Elsevier; Oxford: 1998. p. 149-179.

Kennedy A. Parafoveal processing in word recognition. The Quarterly Journal of Experimental Psychology. 2000; 53(2):429-455. [PubMed: 10881613]

Kennedy A. Parafoveal-on-foveal effects are not an artifact of mislocated saccades. Journal of Eye Movement Research. 2008; 2(1):1-10.

Kennedy A, Pynte J. Parafoveal-on-foveal effects in normal reading. Vision Research. 2005; 45(2): 153-168. [PubMed: 15581917]

Kennedy A, Pynte J, Ducrot S. Parafoveal-on-foveal interactions in word recognition. The Quarterly Journal of Experimental Psychology. 2002; 55(4):1307-1337. [PubMed: 12420997]

Kliegl R. Toward a perceptual-span theory of distributed processing in reading: A reply to Rayner, Pollatsek, Drieghe, Slattery, and Reichle (2007). Journal of Experimental Psychology: General. 2007; 136(3):530-537.

Kliegl R, Engbert R. Fixation durations before word skipping in reading. Psychonomic Bulletin \& Review. 2005; 12(1):132-138. [PubMed: 15945206]

Kliegl R, Masson MEJ, Richter EM. A linear mixed model analysis of masked repetition priming. Visual Cognition. 2010; 18(5):655-681. doi: 10.1080/13506280902986058.

Kliegl R, Risse S, Laubrock J. Preview benefit and parafoveal-on-foveal effects from word n+2. Journal of Experimental Psychology: Human Perception and Performance. 2007; 33(5):12501255. [PubMed: 17924820]

Li X, Bicknell K, Liu P, Wei W, Rayner K. Reading is fundamentally similar across disparate writing systems: A systematic characterization of how words and characters influence eye movements in Chinese reading. Journal of Experimental Psychology: General. 2014; 143(2):895-913. doi: 10.1037/a0033580. [PubMed: 23834023]

Li X, Liu P, Rayner K. Eye movement guidance in Chinese reading: Is there a preferred viewing location. Vision Reserch. 2011; 51:1146-1156.

Li X, Ma G. Word boundaries affect visual attention in Chinese reading. PloS one. 2012; 7(11):e48905. doi: 10.1371/journal.pone.0048905. [PubMed: 23152819]

Li X, Rayner K, Cave K. On the segmentation of Chinese words during reading. Cognitive Psychology. 2009; 58:525-552. [PubMed: 19345938]

Liversedge SP, White SJ, Findlay JM, Rayner K. Binocular coordination of eye movements during reading. Vision Research. 2006; 46:2363-2374. [PubMed: 16540142]

Massidda, D. retimes: Reaction Time Analysis (R package version 0.1-2). 2013. Retrieved from http:// CRAN.R-project.org/package $=$ retimes

McConkie GW, Kerr PW, Reddix MD, Zola D. Eye movement control during reading: I. The location of initial eye fixations on words. Vision Research. 1988; 28(10):1107-1118. [PubMed: 3257013]

Morey, RD.; Rouder, JN. Bayes Factor: Computation of Bayes factors for simple designs (Version 0.9.4) [Computer program]. 2013. Retrieved from http://cran.r-project.org/web/packages/ BayesFactor/

Pollatsek A, Hyönä J. The role of semantic transparency in the processing of Finnish compound words. Language and Cognitive Processes. 2005; 20(1-2):261-290. doi: 10.1080/01690960444000098.

Pynte J, Kennedy A, Ducrot S. The influence of parafoveal typographical errors on eye movements in reading. European Journal of Cognitive Psychology. 2004; 16(1-2):178-202.

R Core Team. R Foundation for Statistical Computing. Vienna, Austria: 2013. R: A language and environment for statistical computing..

Ratcliff R. Group reaction time distributions and an analysis of distribution statistics. Psychological bulletin. 1979; 86(3):446-461. [PubMed: 451109]

Rayner K. Eye movements in reading and information processing: 20 years of research. Psychological Bulletin. 1998; 124(3):372-422. [PubMed: 9849112] 
Rayner K. Eye movements and attention in reading, scene, perception, and visual search. The Quarterly Journal of Experimental Psychology. 2009; 62(8):1457-1506. [PubMed: 19449261]

Rayner K, Fischer MH, Pollatsek A. Unspaced text interferes with both word identification and eye movement control. Vision Research. 1998; 38(8):1129-1144. [PubMed: 9666972]

Rayner K, Li X, Pollatsek A. Extending the E-Z Reader model of eye movement control to Chinese reading. Cognitive Science. 2007; 31:1021-1033. [PubMed: 21635327]

Reichle ED, Drieghe D. Using E-Z Reader to examine the consequences of fixation-location measurement error. Journal of Experimental Psychology: Learning, Memory, and Cognition. 2015; 41(1):262-270. doi: 10.1037/a0037090.

Reichle ED, Liversedge SP, Pollatsek A, Rayner K. Encoding multiple words simultaneously in reading is implausible. Trends in Cognitive Sciences. 2009; 13(3):115-119. doi: 10.1016/j.tics. 2008.12.002. [PubMed: 19223223]

Reichle ED, Pollatsek A, Fisher DL, Rayner K. Toward a model of eye movement control in reading. Psychological Review. 1998; 105(1):125-157. [PubMed: 9450374]

Reichle ED, Rayner K, Pollatsek A. The E-Z Reader model of eye-movement control in reading: Comparisons to other models. Behavioral and Brain Sciences. 2003; 26:445-526. [PubMed: 15067951]

Reichle ED, Warren T, McConnell K. Using E-Z Reader to model the effects of higher level language processing on eye movements during reading. Psychonomic Bulletin \& Review. 2009; 16(1):1-21. [PubMed: 19145006]

Reilly R, Radach R. Some empirical tests of an interactive activation model of eye movement control in reading. Cognitive Systems Research. 2006; 7:34-55.

Risse S, Engbert R, Kliegl R. Eye-movement control in reading: Experimental and corpus-analytic challenges for a computational model. Cognitive and cultural influences on eye movements. 2008:65-91.

Rouder JN, Morey RD, Speckman PL, Province JM. Default Bayes factors for ANOVA designs. Journal of Mathematical Psychology. 2012; 56:356-374.

Rouder JN, Speckman PL, Sun DC, Morey RD, Iverson G. Bayesian t tests for accepting and rejecting the null hypothesis. Psychonomic Bulletin \& Review. 2009; 16(2):225-237. doi: 10.3758/Pbr. 16.2.225. [PubMed: 19293088]

Schotter ER, Angele B, Rayner K. Parafoveal processing in reading. Attention, Perception, \& Psychophysics. 2012; 71(1):5-35.

Starr M, Inhoff A. Attention allocation to the right and left of a fixated word: Use of orthographic information from multiple words during reading. European Journal of Cognitive Psychology. 2004; 16(1-2):203-225.

Staub A, White SJ, Drieghe D, Hollway EC, Rayner K. Distributional effects of word frequency on eye fixation durations. Journal of Experimental Psychology: Human Perception and Performance. 2010; 36(5):1280-1293. [PubMed: 20873939]

van Heuven WJ, Mandera P, Keuleers E, Brysbaert M. SUBTLEX-UK: A new and improved word frequency database for British English. The Quarterly Journal of Experimental Psychology. 2014; 67:1176-1190. [PubMed: 24417251]

Wei W, Li X, Pollatsek A. Word properties of a fixated region affect outgoing saccade length in Chinese. Vision Research. 2013; 80:1-6. [PubMed: 23231957]

White SJ. Eye movement control during reading: Effects of word frequency and orthographic familiarity. Journal of Experimental Psychology: Human Perception and Performance. 2008; 34(1):205-223. [PubMed: 18248149]

White SJ, Staub A. The distribution of fixation durations during reading: Effects of stimulus quality. Journal of Experimental Psychology: Human Perception and Performance. 2012; 38(3):603-617. [PubMed: 21910560]

Yan G, Tian H, Bai X, Rayner K. The effect of word and character frequency on the eye movements of Chinese readers. British Journal of Psychology. 2006; 97:259-268. [PubMed: 16613652]

Yan M, Kliegl R, Shu H, Pan J, Zhou X. Parafoveal load of word N+1 modulates preprocessing effectiveness of word N+2 in Chinese reading. Journal of Experimental Psychology: Human 
Perception and Performance. 2010; 36(6):1669-1676. doi: 10.1037/a0019329. [PubMed: 20731511]

Yan M, Richter EM, Shu H, Kliegl R. Readers of Chinese extract semantic information from parafoveal words. Psychonomic Bulletin \& Review. 2009; 16(3):561-566. [PubMed: 19451385]

Yan M, Sommer W. Parafoveal-on-Foveal effects of emotional word semantics in reading Chinese sentences: Evidence from eye movements. Journal of Experimental Psychology: Learning, Memory, and Cognition. 2015 doi: 10.1037/xlm0000095.

Yan M, Zhou W, Shu H, Kliegl R. Perceptual span depends on font size during the reading of Chinese sentences. Journal of Experimental Psychology: Learning, Memory, and Cognition. 2015; 41(1): 209-219.

Yen M-H, Tsai J-L, Tzeng O-J-L, Hung D-L. Eye movements and parafoveal word processing in reading Chinese. Memory \& Cognition. 2008; 36(5):1033-1045. [PubMed: 18630209]

Yang J, Wang S, Xu Y, Rayner K. Do Chinese readers obtain preview benefit word n+2: Evidence from eye movements. Journal of Experimental Psychology: Human Perception and Performance. 2009; 35(4):1192-1204. [PubMed: 19653758]

Yang JM, Staub A, Li N, Wang S, Rayner K. Plausibility effects when reading one-and two-character words in Chinese: evidence from eye movements. Journal of Experimental Psychology: Learning, Memory, and Cognition. 2012; 38(6):1801-1809.

Zhou W, Kliegl R, Yan M. A validation of parafoveal semantic information extraction in reading Chinese. Journal of Research in Reading. 2013; 36(S1):S51-S63. doi: 10.1111/j.

1467-9817.2013.01556.x. 


\section{Experiment 1A}

HF-C：目前国内对举行国画艺术宣传的重视已超乎人们想象。

(Currently domestic value on propagating Chinese painting is beyond one's

imagination)

LF-C：目前国内对举行油画艺术宣传的重视已超乎人们想象。

(Currently domestic value on propagating oil painting is beyond one's

imagination)

\section{Experiment 1B}

HF-W : 大河东岸的化工厂建造可能引发西岸的生态环境危机。

(Building chemical factory on the east may lead to environment crisis on the west side of the river)

LF-W: 大河东岸的化工厂建造可能引发此岸的生态环境危机。

(Building chemical factory on the east may lead to environment crisis on this side

of the river)

\section{Figure 1.}

Materials used in Experiments 1A and 1B. The target words are in bold and the first character is underlined for the purpose of illustration (characters were not in bold or underlined in the experiment).

J Exp Psychol Hum Percept Perform. Author manuscript; available in PMC 2016 October 01. 


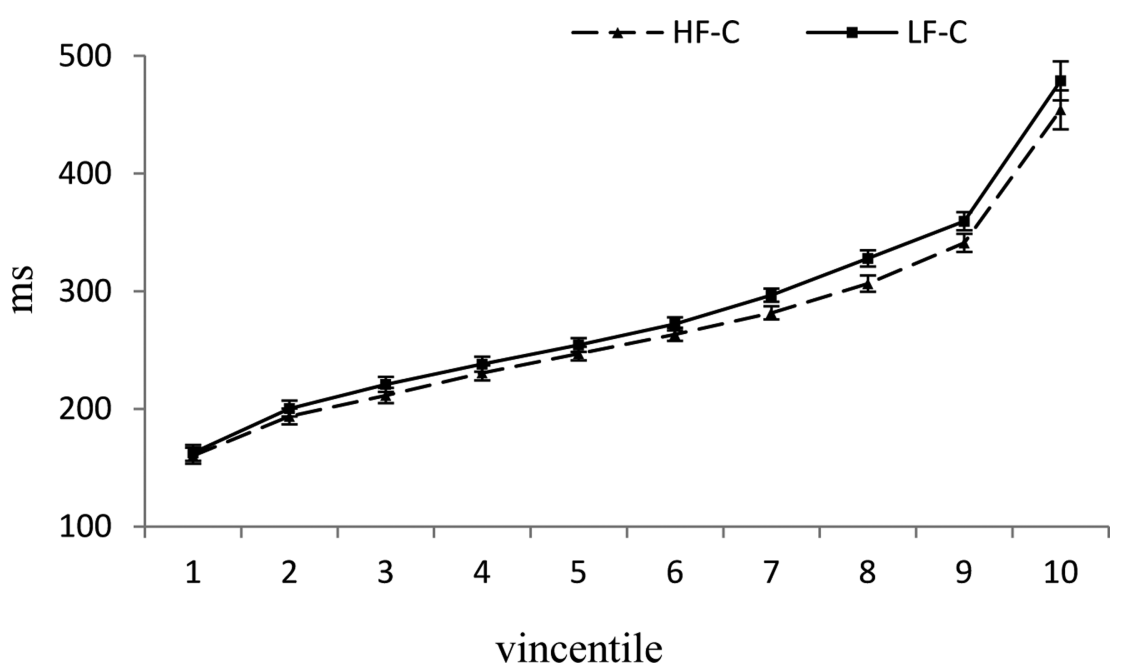

Figure 2.

Vincentile plot for the high-frequency character (HF-C) and low-frequency character (LF-C) conditions in Experiment 1A. 


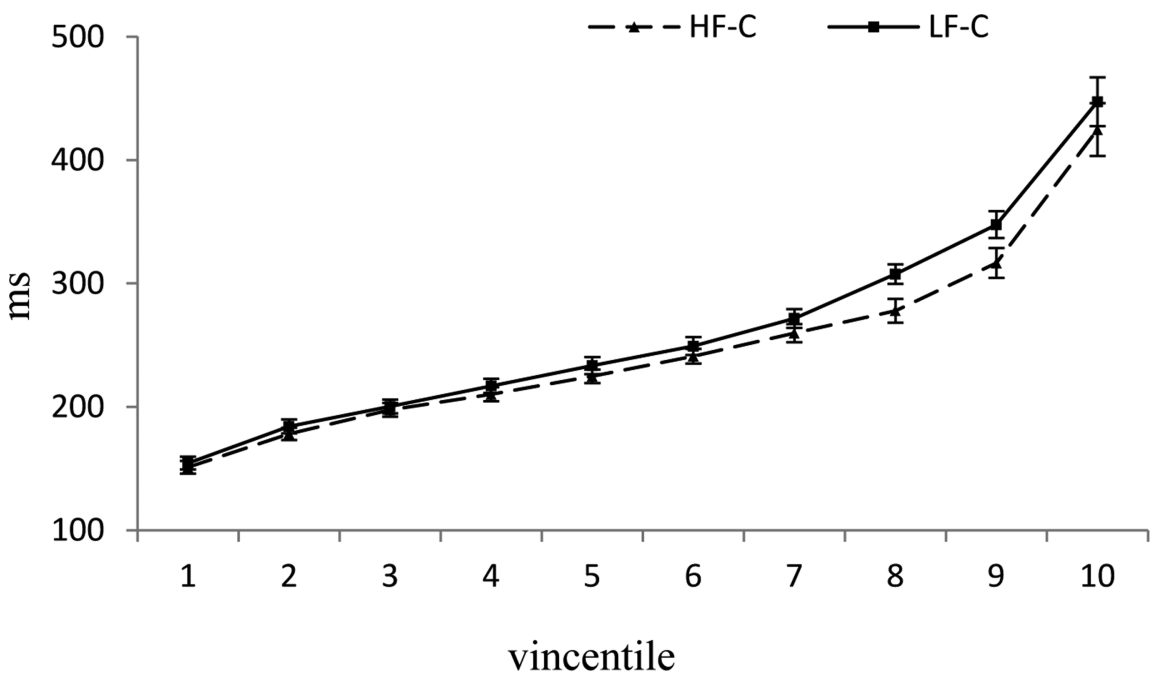

Figure 3.

Vincentile plot for the high-frequency character (HF-C) and low-frequency character (LF-C) conditions in Experiment 2A. 


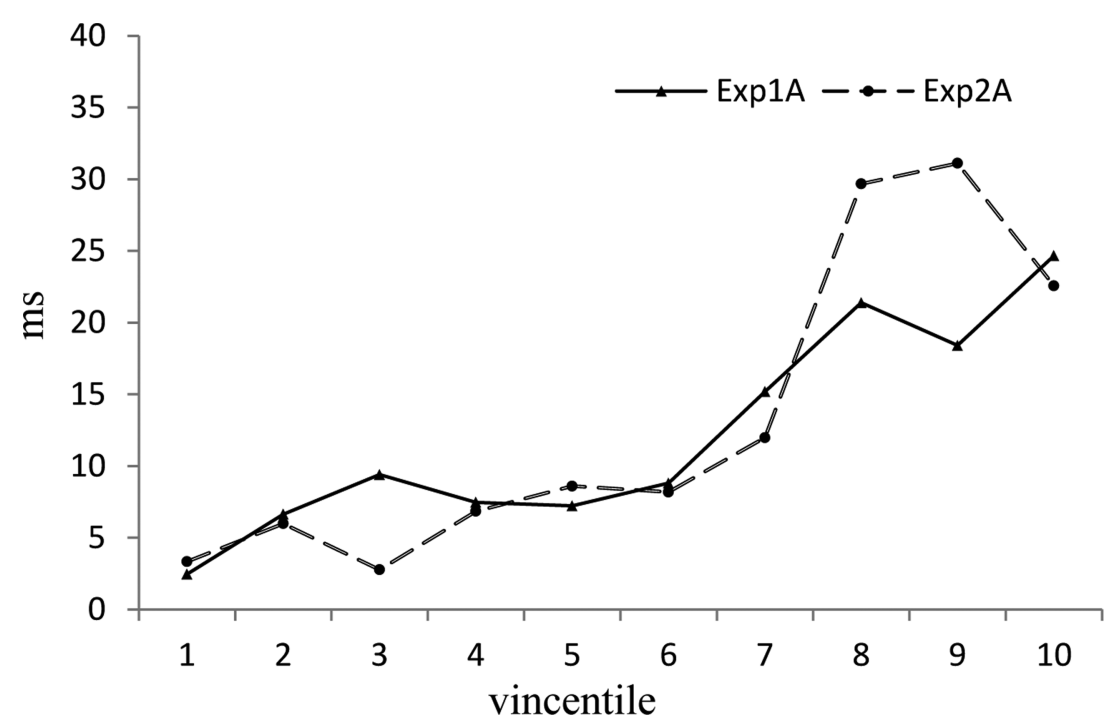

Figure 4.

Mean differences between the high-frequency character (HF-C) and low-frequency character (LF-C) conditions in Experiments 1A and 2A. 